\title{
Living alone and cardiovascular disease outcomes
}

\author{
Sumeet Gandhi, ${ }^{1}$ Shaun G Goodman, ${ }^{1}$ Nicola Greenlaw, ${ }^{2}$ Ian Ford, ${ }^{2}$ \\ Paula McSkimming, ${ }^{2}$ Roberto Ferrari, ${ }^{3}$ Yangsoo Jang, ${ }^{4}$ Marco Antonio Alcocer-Gamba, ${ }^{5}$ \\ Kim Fox, ${ }^{6}$ Jean-Claude Tardif, ${ }^{7}$ Michal Tendera, ${ }^{8}$ Paul Dorian, ${ }^{1}$ Gabriel Steg, ${ }^{6,9}$ \\ Jacob Allan Udell ${ }^{10}$
}

- Additional material is published online only. To view please visit the journal online (http://dx.doi.org/10.1136/ heartjnl-2018-313844)

For numbered affiliations see end of article.

Correspondence to Jacob Allan Udell, Cardiovascular Division Department of Medicine Women's College Hospital, Women's College Research Institute, University of Toronto, Toronto ON M5S 1B1, Canada; jay.udell@utoronto.ca

Received 20 July 2018 Revised 9 January 2019 Accepted 14 January 2019 Published Online First 21 February 2019

\section{ABSTRACT \\ Objective To evaluate cardiovascular (CV) outcomes in outpatients with coronary artery disease (CAD) living alone compared with those living with others. \\ Methods The prospeCtive observational LongitudinAl Reglstry of patients with stable coronarY artery disease (CLARIFY) included outpatients with stable CAD. CLARIFY enrolled participants in 45 countries from November 2009 to July 2010, with 5 years of follow-up. Living arrangement was documented at baseline. The primary outcome was a composite of major adverse cardiovascular events (MACEs) defined as CV death, myocardial infarction (MI) and stroke. \\ Results Among 32367 patients, 3648 patients were} living alone (11.3\%). After multivariate adjustment, there were no residual differences in MACE among patients living alone compared with those living with others (HR $1.04,95 \% \mathrm{Cl} 0.92$ to $1.18, p=0.52)$; however, there was significant heterogeneity in the exposure effect by sex $\left(P_{\text {interaction }}<0.01\right)$. Specifically, men living alone were at higher risk for MACE (HR 1.17, 95\% Cl 1.002 to 1.36, $\mathrm{p}=0.047$ ) as opposed to women living alone (HR 0.82, $95 \% \mathrm{Cl} 0.65$ to $1.04, \mathrm{p}=0.1)$, predominantly driven by a heterogeneous effect by sex on $\mathrm{MI}\left(\mathrm{P}_{\text {interaction }}=0.006\right)$. There was no effect modification for MACE by age group $\left(P_{\text {interaction }}=0.3\right)$, although potential varying effects by age for Ml $\left(P_{\text {interaction }}=0.046\right)$ and stroke $\left(P_{\text {interaction }}=0.05\right)$.

Conclusions Living alone was not associated with an independent increase in MACE, although significant sexbased differences were apparent. Men living alone may have a worse prognosis from CV disease than women; further analyses are needed to elucidate the mechanisms underlying this difference.

Trial registration number ISRCTN43070564.

\section{INTRODUCTION}

Social isolation refers to the lack of contact an individual has with society, with living alone frequently used as a surrogate. ${ }^{1}$ Living alone may lead to poor outcomes in patients with coronary artery disease (CAD) through a complex interaction between increased neurohormonal stress with accelerated atherosclerosis, less adherence to guideline recommended therapy and secondary prevention targets, increased anxiety and depression leading to more psychological distress, poor coping mechanisms/ self-care and less access to healthcare services. ${ }^{2} 3$ Previous analyses have sought to gain insight into the risk of living alone in patients with established cardiovascular disease (CVD). The effect of living alone has been variable due to the significant heterogeneity among the populations studied, with subgroup analysis showing potential effect modification by age and sex, and potential lower risk among women and elderly patients living alone. ${ }^{4-7}$

Patients with CAD are diverse with differences in ethnicity, socioeconomic status, location and psychosocial factors that may play a role in the risk of recurrent cardiovascular (CV) events. ${ }^{8}$ The prospeCtive observational LongitudinAl RegIstry oF patients with stable coronarY artery disease (CLARIFY) was initiated to improve knowledge about patients with stable CAD from a broad geographic perspective. ${ }^{9}$ As individuals with stable CAD live longer with advanced comorbidities, there is an important public health implication to determine if living status is independently associated with poor health outcomes. The objective of this analysis was to determine, in a stable CAD population, if living alone is associated with increased CV risk. Given previous research, effect modification by sex and age group were further explored in this post hoc analysis.

\section{METHODS}

\section{Study design and patient selection}

The CLARIFY study cohort included outpatients with stable CAD with 5 years of follow-up; the study methods and design were previously published. $^{9-12}$ Patients eligible for enrolment were those with stable CAD diagnosed by at least one of the following: (1) documented myocardial infarction (MI) (>3 months ago); (2) coronary stenosis $>50 \%$ on coronary angiography; (3) chest pain with myocardial ischaemia determined by stress ECG, stress echocardiography or myocardial imaging; or (4) history of revascularisation by coronary artery bypass graft (CABG) surgery or percutaneous coronary intervention (PCI) (performed $>3$ months ago). Patients hospitalised for CVD within the previous 3 months (including for revascularisation), patients for whom revascularisation was planned and patients with conditions expected to hamper participation of 5-year follow-up were excluded from participating in the study. A total of 32703 subjects were enrolled in 45 countries from November 2009 to July 2010 (see online supplementary table 1 ).

\section{Data collection}

The investigators completed standardised electronic case report forms at baseline and yearly 
at each visit for up to 5 years. Living arrangement status was documented as either 'living alone' or 'not living alone' at baseline. Further information collected at baseline included demographics; medical history and CV risk factors; current symptoms; physical examination; laboratory values (eg, fasting blood glucose, haemoglobin A1c, cholesterol and triglycerides); and current chronic drugs regimen (ie, those taken regularly by the patient for $\geq 7$ days before entry in the registry). Data were recorded if an ECG was available for whether the patient was in sinus rhythm, atrial fibrillation/atrial flutter, paced rhythm or left bundle branch block. The remaining patients may not have had ECG data available or may be in another rhythm then above. Investigators reported number of vessels with disease and number of coronary territories with significant stenosis $(>50 \%)$ independent of whether the patient had a recent angiogram (within 12 months). These data were investigator reported independent of the most recent available angiogram results and may not be mutually exclusive.

For patients missing the yearly in-person visit, telephone contact with the patient, a designated relative or contact or their physician was attempted. To ensure data quality, onsite-monitoring visits of $100 \%$ of the data in $5 \%$ of centres were selected at random, regular telephone contact with investigators to reduce missing data and loss to follow-up, and centralised verification of the electronic case report forms for completeness, consistency and accuracy were undertaken.

\section{Outcomes}

At each annual follow-up visit, clinical outcomes occurring during the previous 12 months were recorded. The primary outcome of this analysis was major adverse cardiovascular events (MACE), which included CV death, non-fatal or fatal MI and non-fatal or fatal stroke. Secondary outcomes were all-cause death, CV death, MI, stroke, unstable angina and major bleeding (defined as leading to hospitalisation or transfusion). CV death was defined as fatal MI or stroke, other CV death or death due to unknown cause; any MI or stroke followed by death in the subsequent 28 days was considered fatal. Events were accepted as reported by patients and physicians, without central adjudication; however, all events were source verified during audits.

The study was performed in accordance with the principles of the Declaration of Helsinki. Approval was also obtained in all participating countries, in accordance with local regulations before recruitment of the first participant. All patients gave written informed consent to participate, in accordance with national and local guidelines. There was no patient/public involvement in this research study. CLARIFY is registered in the ISRCTN registry of clinical trials.

\section{Statistical analysis}

All CLARIFY data were collected and analysed at an independent academic statistics centre at the Robertson Centre for Biostatistics, University of Glasgow, UK, which was responsible for managing the database, performing all analyses and data storage. Baseline variables are summarised as means and SDs or medians and IQRs for continuous data, depending on the distribution of the data, and as counts and percentages for categorical data. In this post hoc analysis, differences between patients living alone and those living with others were compared using $\chi^{2}$ tests or Fisher's exact test for categorical variables as appropriate, and two sample t-tests or Wilcoxon-Mann-Whitney tests for continuous variables, depending on the distribution of the data. A Cox proportional hazards model was used to assess the risk associated with living alone and time to first $\mathrm{CV}$ outcomes. Crude and multivariable adjusted HRs and corresponding 95\% CIs were estimated after adjustment for age, sex and geographic region, as well as baseline history of smoking, diabetes, peripheral arterial disease, MI, PCI, CABG, asthma/chronic obstructive pulmonary disease and congestive heart failure (CHF). Additional clinical characteristics were also adjusted for, including baseline systolic blood pressure, diastolic blood pressure, left ventricular ejection fraction and number of vessels with coronary artery stenoses. Heterogeneity was assessed with interaction testing between living status and sex, age group $(<65$ years, $65-74$ years and $\geq 75$ years) and history of prior MI at baseline for primary and secondary endpoints after multivariable adjustment. All $\mathrm{p}$ values for the Cox proportional hazards models were obtained using the Wald test.

\section{RESULTS}

A total of 32367 patients were eligible for inclusion in the analysis with 3648 patients documented as living alone (11.3\%) and $28728(88.8 \%)$ patients living with others. Tables 1 and 2 include baseline characteristics, study inclusion, medical history, $\mathrm{CV}$ risk factors and symptom profile. The mean age for patients living alone was $67( \pm 10.66)$ years. Patients living alone were older, more likely to be female, predominantly white, less likely to be employed full time and more likely to be retired than those living with others. More patients living alone were current smokers, had a history of atrial fibrillation, prior HF hospitalisation, history of peripheral arterial disease and yet had less diabetes. Table 3 describes CV therapies. There was a high use of guideline recommended pharmacological therapy in both groups; there was a lower number of patients living alone taking thienopyridines, beta-blockers and statins.

In unadjusted models, patients living alone had a higher risk of the primary endpoint of MACE $(10.3 \%$ vs $8.5 \%$, HR 1.24 , $95 \%$ CI 1.11 to $1.38, \mathrm{p}<0.001)$, all-cause death $(9.8 \%$ vs $7.6 \%$, HR $1.31,95 \%$ CI 1.17 to $1.47, \mathrm{p}<0.001)$, CV death $(6.5 \%$ vs $4.8 \%$, HR $1.37,95 \% \mathrm{CI} 1.20$ to $1.58, \mathrm{p}<0.001)$ and stroke ( $2.7 \%$ vs $2.0 \%$, HR $1.35,95 \%$ CI 1.09 to $1.67, \mathrm{p}=0.006$ ). There was no difference in rates of MI, unstable angina, major bleeding or hospitalisation for CHF (table 4). After adjustment for age and sex, there remained statistically significant differences in the all-cause and CV death outcomes; following additional multivariate adjustment, there were no residual differences in outcomes in patients living alone compared with those living with others (table 4).

Nevertheless, there was significant heterogeneity identified in the exposure effect by sex $\left(\mathrm{P}_{\text {interaction }}<0.01\right)$ (figure 1). Specifically, men living alone were at higher risk for MACE (HR 1.17, $95 \%$ CI 1.002 to $1.36, p=0.047$ ) as opposed to women living alone (HR 0.82, 95\% CI 0.65 to 1.04, $\mathrm{p}=0.099$ ). This difference was primarily driven by effect modification by sex for $\mathrm{MI}\left(\mathrm{P}_{\text {inte }}\right.$

$=0.006)$ with no difference in $\mathrm{CV}$ death $\left(\mathrm{P}_{\text {interaction }}=0.075\right)$ and stroke $\left(\mathrm{P}_{\text {interaction }}=0.99\right)$. Women living alone in comparison with women living with others showed a significantly lower adjusted risk of MI $(2.2 \%$ vs $3.2 \%$, HR $0.54,95 \%$ CI 0.35 to $0.84, \mathrm{p}=0.007)$ that was not apparent in men $(4.0 \%$ vs $3.2 \%$, HR $1.17,95 \%$ CI 0.92 to $1.49, \mathrm{p}=0.19$ ).

The effect of living alone for MACE was consistent across age groups ( $<65$ years, 65-74 years and $\geq 75$ years, $\mathrm{P}_{\text {interaction }}=0.33$ ). There were potential varying effects of living alone by age group for MI $\left(\mathrm{P}_{\text {interaction }}=0.046\right)$ and stroke $\left(\mathrm{P}_{\text {interaction }}=0.05\right)$ (figure 2$)$. Specifically, the risk of MI tended to be lowest among older patients ( $\geq 75$ years) living alone in comparison with older 
Table 1 Baseline characteristics by living arrangement status

\begin{tabular}{|c|c|c|c|}
\hline & $\begin{array}{l}\text { Living alone } \\
(\mathrm{n}=3648)\end{array}$ & $\begin{array}{l}\text { Not living alone } \\
(\mathrm{n}=28728)\end{array}$ & $P$ value \\
\hline Age (years), mean (SD) & $67.2(10.7)$ & $63.8(10.4)$ & $<0.001$ \\
\hline Males (\%) & $2254(61.8)$ & $22857(79.6)$ & $<0.001$ \\
\hline $\begin{array}{l}\text { Body mass index }(\mathrm{kg}) \text {, median } \\
\text { (25th, } 75 \text { th quartiles), }\end{array}$ & $27(25,31)$ & $27(25,30)$ & 0.032 \\
\hline $\begin{array}{l}\text { Waist circumference }(\mathrm{cm}) \text {, median } \\
\text { (25th, 75th quartiles) }\end{array}$ & $96(88,105)$ & $97(89,105)$ & 0.01 \\
\hline \multicolumn{4}{|l|}{ Ethnicity, n (\%) } \\
\hline White & $2644(72.5)$ & $18304(63.8)$ & $<0.001$ \\
\hline South Asian & $130(3.6)$ & $2285(8.0)$ & \\
\hline Chinese & $167(4.6)$ & $2573(9.0)$ & \\
\hline Japanese/Korean & $142(3.9)$ & $893(3.1)$ & \\
\hline Hispanic & $110(3.0)$ & $1458(5.1)$ & \\
\hline Black/African & $44(1.2)$ & $294(1.0)$ & \\
\hline Unknown & $411(11.3)$ & $2921(10.2)$ & \\
\hline \multicolumn{4}{|l|}{ Employment status, n (\%) } \\
\hline Employed full-time & $605(16.6)$ & $7291(25.4)$ & $<0.001$ \\
\hline Employed part-time & $227(6.2)$ & $2022(7.0)$ & \\
\hline Unable to work & $146(4.0)$ & $1119(3.9)$ & \\
\hline Unemployed & $143(3.9)$ & $1689(5.9)$ & \\
\hline Retired & $2415(66.2)$ & $15505(54.0)$ & \\
\hline Other & $112(3.1)$ & $1101(3.8)$ & \\
\hline \multicolumn{4}{|l|}{ Education level, n (\%) } \\
\hline Primary school (or less) & $1011(27.7)$ & $7561(26.3)$ & $<0.001$ \\
\hline Secondary school & $1785(49.0)$ & $13251(46.1)$ & \\
\hline College/university & $852(23.4)$ & 7913 (27.6) & \\
\hline \multicolumn{4}{|l|}{ Medical history, n (\%) } \\
\hline Myocardial infarction & $2150(59.0)$ & $17251(60.0)$ & 0.20 \\
\hline $\mathrm{PCl}$ & $2051(56.2)$ & $16906(58.9)$ & 0.003 \\
\hline CABG & $907(24.9)$ & $6722(23.4)$ & 0.049 \\
\hline Hospitalisation for CHF & $218(6.0)$ & $1294(4.5)$ & $<0.001$ \\
\hline Internal cardiac defibrillator & $65(1.8)$ & $342(1.2)$ & 0.003 \\
\hline Pacemaker & $124(3.4)$ & $653(2.3)$ & $<0.001$ \\
\hline Aortic abdominal aneurysm & $70(1.9)$ & $424(1.5)$ & 0.040 \\
\hline Carotid disease & $350(9.6)$ & $2107(7.3)$ & $<0.001$ \\
\hline Peripheral arterial disease & $425(11.7)$ & $2777(9.7)$ & $<0.001$ \\
\hline Transient ischaemic attack & $137(3.8)$ & $856(3.0)$ & 0.010 \\
\hline Stroke & $166(4.6)$ & $1135(4.0)$ & 0.082 \\
\hline Atrial fibrillation/flutter & $324(8.9)$ & $1960(6.8)$ & $<0.001$ \\
\hline Family history of premature CAD & $1120(30.7)$ & $8096(28.2)$ & 0.001 \\
\hline Treated hypertension & $2638(72.3)$ & $20351(70.9)$ & 0.066 \\
\hline Diabetes & $977(26.8)$ & $8415(29.3)$ & 0.002 \\
\hline Dyslipidaemia & $2773(76.0)$ & $21485(74.8)$ & 0.109 \\
\hline Asthma/COPD & $363(10.0)$ & $2031(7.1)$ & $<0.001$ \\
\hline \multicolumn{4}{|l|}{ Smoking status, n (\%) } \\
\hline Current & $536(14.7)$ & $3501(12.2)$ & $<0.001$ \\
\hline Former & $1505(41.3)$ & $13468(46.9)$ & \\
\hline Never & $1607(44.0)$ & $11759(40.9)$ & \\
\hline \multicolumn{4}{|c|}{ Alcohol intake (number of drinks per week), $n(\%)$} \\
\hline 0 & $1741(47.7)$ & $13684(47.6)$ & 0.032 \\
\hline$>0$ and $<20$ & $1745(47.8)$ & $14025(48.8)$ & \\
\hline $20-40$ & $144(4.0)$ & $921(3.2)$ & \\
\hline$>40$ & $18(0.5)$ & $93(0.3)$ & \\
\hline \multicolumn{4}{|l|}{ Stimulant drinks consumed, $\mathrm{n}(\%)$} \\
\hline Coffee & $1789(49.1)$ & $13551(47.2)$ & $<0.001$ \\
\hline Tea & $1200(32.9)$ & $8781(30.6)$ & \\
\hline Neither & $658(18.0)$ & $6380(22.2)$ & \\
\hline
\end{tabular}

Continued

\begin{tabular}{lllc}
\hline Table 1 Continued & \multicolumn{1}{c}{ Living alone } \\
& $(\mathbf{n = 3 6 4 8 )}$ & $\begin{array}{l}\text { Not living alone } \\
(\mathbf{n}=28728)\end{array}$ & P value \\
\hline $\begin{array}{l}\text { Daily intake of stimulant drinks } \\
\text { (cups/day), median (25th, 75th } \\
\text { quartiles) }\end{array}$ & $2(2,4)$ & $2(2,4)$ & 0.011 \\
$\begin{array}{l}\text { Physical activity, } \mathrm{n}(\%) \\
\text { No physical activity weekly }\end{array}$ & $639(17.5)$ & $4584(16.0)$ & 0.023 \\
$\begin{array}{l}\text { Light physical activity most } \\
\text { weeks }\end{array}$ & $1850(50.7)$ & $14782(51.5)$ & \\
$\begin{array}{l}\text { At least } 20 \text { min vigorous physical } \\
\text { activity once or twice a week }\end{array}$ & $567(15.6)$ & $4860(16.9)$ & \\
$\begin{array}{l}\text { At least 20 min vigorous physical } \\
\text { activity at least three times a } \\
\text { week }\end{array}$ & $591(16.2)$ & $4495(15.7)$ & \\
\hline
\end{tabular}

CABG, coronary artery bypass graft surgery; $C A D$, coronary artery disease; $C H F$, congestive heart failure; $\mathrm{COPD}$, chronic obstructive pulmonary disease; $\mathrm{PCl}$, percutaneous coronary intervention.

patients living with others $(3.3 \%$ vs $4.2 \%$, HR $0.67,95 \% \mathrm{CI}$ 0.43 to $1.04, p=0.077)$, intermediate risk among middle-aged (65-74 years) patients $(2.9 \%$ vs $3.4 \%$, HR $0.83,95 \%$ CI 0.57 to $1.21, \mathrm{p}=0.33)$ and highest among younger $(<65$ years) patients in the cohort $(3.9 \%$ vs $2.9 \%$, HR $1.31,95 \%$ CI 0.96 to 1.78 , $\mathrm{p}=0.088$ ). Similar findings were observed for stroke (figure 2). The effect of living alone was consistent in patients with and without prior MI $\left(\mathrm{P}_{\text {interaction }}=0.44\right)$.

\section{DISCUSSION}

CLARIFY is the largest international registry to describe the association of living alone with $\mathrm{CV}$ outcomes in a contemporary population of patients with stable CAD. Despite an indication that people living alone had a higher incidence of several outcomes in unadjusted analyses including the primary endpoint of MACE, the results of the adjusted analysis revealed no independent risk of MACE in patients living alone. A novel aspect to our analysis is that we did observe potential sex-based heterogeneity for the effects of living alone. Specifically, women living alone had a trend towards lower risk of MI compared with women or men living with others. Older patients living alone demonstrated a trend towards a lower risk of MI and stroke, while the trends reversed for younger patients living alone.

Recent studies have described the association of living alone with adverse events in patients with CVD. The Coronary Revascularization Demonstrating Outcome Study in Kyoto Acute Myocardial Infarction registry included patients with acute coronary syndrome (ACS) who underwent PCI; at 5-year follow-up, patients living alone did not have an increased risk of death or $\mathrm{CV}$ events but an increased risk of HF admission. Their study did not show heterogeneity in patients $\geq 75$ years. $^{6}$ In patients post-ACS from Japan, The Osaka Acute Coronary Insufficiency Study registry showed patients living alone had overall increased MACE, with female patients living alone showing a trend towards increased risk. ${ }^{13}$ The Reduction of Atherothrombosis for Continued Health (REACH) registry was a large, prospective, observational registry with $>40000$ patients with high vascular risk and established CVD. Their results showed that living alone was associated with an increased risk of 4-year mortality and CV death in patients with established $\mathrm{CVD}^{5}$; there was heterogeneity in risk of events according to age with lower risk in elderly patients $>80$ years old. In contrast to REACH, our analysis did not show a difference in all-cause and CV death according to living situation, potentially due to several reasons. Compared 
Table 2 Baseline cardiovascular characteristics by living arrangement status

\begin{tabular}{|c|c|c|c|}
\hline & $\begin{array}{l}\text { Living alone } \\
(\mathrm{n}=3648)\end{array}$ & $\begin{array}{l}\text { Not living alone } \\
(n=28728)\end{array}$ & $P$ value \\
\hline Any angina, n (\%) & $840(23.0)$ & $6328(22.0)$ & 0.17 \\
\hline Angina and CCS class & & & 0.13 \\
\hline No angina & $2807(77.0)$ & $22399(78.0)$ & \\
\hline Angina CCS class I & $263(7.2)$ & $1788(6.2)$ & \\
\hline Angina CCS class II & $417(11.4)$ & $3396(11.8)$ & \\
\hline Angina CCS class III & $150(4.1)$ & $1075(3.7)$ & \\
\hline Angina CCS class IV & $10(0.3)$ & $68(0.2)$ & \\
\hline $\begin{array}{l}\text { CHF symptoms including NYHA } \\
\text { class, }(\%)\end{array}$ & & & 0.89 \\
\hline No CHF & $3098(84.9)$ & $24375(84.9)$ & \\
\hline CHF NYHA class II & $456(12.5)$ & $3642(12.7)$ & \\
\hline CHF NYHA class III & $94(2.6)$ & $709(2.5)$ & \\
\hline Heart rate (palpation), mean & $68.3(10.6)$ & $68.2(10.6)$ & 0.79 \\
\hline $\mathrm{SBP}(\mathrm{mm} \mathrm{Hg})$, mean & $132.2(17.2)$ & $130.9(16.6)$ & $<0.001$ \\
\hline $\mathrm{DBP}(\mathrm{mm} \mathrm{Hg})$, mean & $76.9(10.1)$ & $77.3(10.0)$ & 0.020 \\
\hline $\begin{array}{l}\text { Left ventricular ejection fraction (\%), } \\
\text { mean }\end{array}$ & $56.5(11.3)$ & $56.0(11.0)$ & 0.032 \\
\hline $\begin{array}{l}\text { Number of vessels with disease, } \\
\mathrm{n}(\%)^{*}\end{array}$ & & & 0.005 \\
\hline 0 & $139(4.6)$ & $864(3.5)$ & \\
\hline 1 & $1279(41.9)$ & $10053(40.9)$ & \\
\hline 2 or more & $1634(53.5)$ & $13649(55.6)$ & \\
\hline $\begin{array}{l}\text { Coronary territories with stenosis } \\
>50 \%, \mathrm{n}(\%)^{*}\end{array}$ & & & \\
\hline Left main & $335(9.2)$ & $2500(8.7)$ & 0.33 \\
\hline Left anterior descending & $2075(56.9)$ & $16827(58.6)$ & 0.051 \\
\hline Circumflex artery & $1208(33.1)$ & $10468(36.5)$ & $<0.001$ \\
\hline Right coronary artery & $1520(41.7)$ & $12590(43.8)$ & 0.013 \\
\hline Bypass graft & $299(8.2)$ & $2300(8.0)$ & 0.69 \\
\hline No significant stenosis & $146(4.0)$ & $906(3.2)$ & 0.007 \\
\hline $\begin{array}{l}\text { Coronary angiography not } \\
\text { performed (within } 12 \text { months) }\end{array}$ & $586(16.1)$ & $4138(14.4)$ & 0.007 \\
\hline ECG rhythm, n (\%)† & & & $<0.001$ \\
\hline Sinus rhythm & $2462(93.1)$ & $20499(95.2)$ & \\
\hline Atrial fibrillation/flutter & $121(4.6)$ & $702(3.3)$ & \\
\hline Paced rhythm & $62(2.3)$ & $332(1.5)$ & \\
\hline LBBB & $158(6.0)$ & $1025(4.8)$ & 0.0063 \\
\hline $\mathrm{HbA1C}(\%)$, mean (SD) & $6.7(1.2)$ & $6.8(1.8)$ & 0.018 \\
\hline $\begin{array}{l}\text { Creatinine (mmol/L), median ( } 25 \text { th, } \\
\text { 75th quartiles) }\end{array}$ & $87(74,100)$ & $88(76,102)$ & $<0.001$ \\
\hline $\begin{array}{l}\text { Haemoglobin }(\mathrm{g} / \mathrm{dL}) \text {, median ( } 25 \text { th, } \\
75 \text { th quartiles) }\end{array}$ & $13.9(12.9,14.9)$ & $14.1(13.0,15.0)$ & $<0.001$ \\
\hline $\begin{array}{l}\text { Fasting blood glucose (mmol/L), } \\
\text { median ( } 25 \text { th, } 75 \text { th quartiles) }\end{array}$ & $5.6(5.0,6.5)$ & $5.7(5.1,6.7)$ & $<0.001$ \\
\hline $\begin{array}{l}\text { Total cholesterol (mmol/L), median } \\
\text { (25th, } 75 \text { th quartiles) }\end{array}$ & $4.4(3.7,5.1)$ & $4.3(3.7,5.0)$ & 0.002 \\
\hline $\begin{array}{l}\mathrm{HDL} \text { (mmol/L), median (25th, 75th } \\
\text { quartiles) }\end{array}$ & $1.2(1.0,1.5)$ & $1.1(1.0,1.4)$ & $<0.001$ \\
\hline $\begin{array}{l}\mathrm{LDL} \text { (mmol/L), median (25th, } 75 \text { th } \\
\text { quartiles) }\end{array}$ & $2.37(1.89,2.94)$ & $2.37(1.90,2.94)$ & 0.68 \\
\hline $\begin{array}{l}\text { Fasting triglycerides (mmol/L), median } \\
\text { (25th, 75th quartiles) }\end{array}$ & $1.39(1.00,1.88)$ & $1.40(1.02,1.93)$ & 0.15 \\
\hline \multicolumn{4}{|c|}{$\begin{array}{l}\text { *Investigators reported number of vessels with disease and number of coronary territories } \\
\text { with significant stenosis ( }>50 \% \text { ) independent of whether the patient had a recent } \\
\text { angiogram (within } 12 \text { months). These data were investigator reported independent of the } \\
\text { most recent available angiogram results and may not be mutually exclusive. } \\
\text { tData were recorded if an ECG was available for whether the patient was in sinus rhythm, } \\
\text { atrial fibrillation/atrial flutter, paced rhythm or LBBB. The remaining patients may not have } \\
\text { had ECG data available or may be in another rhythm then above. } \\
\text { CCS, Canadian Cardiovascular Society; CHF, congestive heart failure; DBP, diastolic blood } \\
\text { pressure; HbA1C, haemoglobin A1C; HDL, high-density lipoprotein; LBBB, left bundle branch } \\
\text { block; LDL, low-density lipoprotein; NYHA, New York Heat Association; SBP, systolic blood } \\
\text { pressure. }\end{array}$} \\
\hline
\end{tabular}

Table 3 Medications and reimbursement status at baseline by living arrangement status

\begin{tabular}{|c|c|c|c|}
\hline & $\begin{array}{l}\text { Living alone } \\
(\mathrm{n}=3648)\end{array}$ & $\begin{array}{l}\text { Not living alone } \\
(\mathrm{n}=28728)\end{array}$ & $P$ value \\
\hline \multicolumn{4}{|l|}{ Medication, n (\%) } \\
\hline Aspirin & $3154(86.5)$ & $25257(87.9)$ & 0.011 \\
\hline Thienopyridine & 835 (22.9) & $7944(27.7)$ & $<0.001$ \\
\hline Other antiplatelets & $291(8.0)$ & $2695(9.4)$ & 0.006 \\
\hline$\geq 2$ antiplatelets & $3644(99.9)$ & $28721(99.9)$ & $<0.001$ \\
\hline Oral anticoagulants & $299(8.2)$ & $2331(8.1)$ & 0.87 \\
\hline Beta-blockers & $2652(72.7)$ & $21718(75.6)$ & $<0.001$ \\
\hline $\begin{array}{l}\text { Symptoms indicative of intolerance } \\
\text { or contraindication to beta-blockers }\end{array}$ & $516(14.2)$ & $4167(14.5)$ & 0.56 \\
\hline Ivabradine & $330(9.1)$ & $2873(10.0)$ & 0.069 \\
\hline Calcium antagonists & $1057(29.0)$ & $7765(27.0)$ & 0.012 \\
\hline ACE inhibitor or ARB & $2790(76.5)$ & $21902(76.2)$ & 0.73 \\
\hline Lipid-lowering drugs & $3301(90.5)$ & $26598(92.6)$ & $<0.001$ \\
\hline Statins & $2954(81.0)$ & $23886(83.2)$ & 0.001 \\
\hline Long-acting nitrates & $764(21.0)$ & $6313(22.0)$ & 0.16 \\
\hline Other antianginal agents & $491(13.5)$ & $4030(14.0)$ & 0.36 \\
\hline Diuretics & $3154(86.5)$ & $25257(88.0)$ & 0.011 \\
\hline Other antihypertensive agents & $283(7.8)$ & $1946(6.8)$ & 0.027 \\
\hline Digoxin and derivatives & $108(3.0)$ & $706(2.5)$ & 0.067 \\
\hline Amiodarone/dronedarone & $111(3.0)$ & $835(2.9)$ & 0.64 \\
\hline Other antiarrhythmics & $46(1.3)$ & $260(0.9)$ & 0.036 \\
\hline NSAIDs & $237(6.5)$ & $1350(4.7)$ & $<0.001$ \\
\hline Antidiabetic agents & $802(23.0)$ & $7126(24.8)$ & $<0.001$ \\
\hline Proton-pump inhibitors & $987(27.1)$ & $7029(24.5)$ & $<0.001$ \\
\hline Thyroid HRT & $263(7.2)$ & $1144(4.0)$ & $<0.001$ \\
\hline HRT in postmenopausal women & $16(0.4)$ & $82(0.3)$ & 0.11 \\
\hline Erectile dysfunction & $60(2.7)$ & $459(2.0)$ & 0.04 \\
\hline $\begin{array}{l}\text { Reimbursement of cardiovascular } \\
\text { agents, } n(\%)\end{array}$ & & & $<0.001$ \\
\hline Fully reimbursed & $1612(44.3)$ & $11044(38.5)$ & \\
\hline Partly reimbursed & $1363(37.5)$ & $10880(37.9)$ & \\
\hline Not reimbursed & $665(18.3)$ & 6786 (23.6) & \\
\hline
\end{tabular}

with REACH, patients included in the CLARIFY registry were younger, had less conventional risk factors and prior history of vascular disease and represented a lower risk population evident by the lower event rates for death and CV outcomes. Patients in the CLARIFY registry also were on more guideline-recommended secondary preventative therapy.

The potential sex-specific differences in CV outcomes according to living situation supports previous literature exploring this association (table 5). ${ }^{5714-20}$

There are several mechanisms that may account for the discrepancy in CV events seen between men and women living alone. Historically, women manage the household and assume a nurturing role and may develop superior self-care skills than their male counterparts. Women socialise differently than men and may form stronger social networks outside of their cohabitation, relying less on spousal support compared with men. ${ }^{21}$ Men living alone who were previously cohabitating with women may not have developed adequate independent coping mechanisms and social supports. This may lead to poor outreach with less attendance to physician appointments or cardiac rehabilitation and seeking out medical attention when necessary. ${ }^{19}$ Previous studies have shown heterogeneity between men and women in regards to stressful live events and 
Table 4 Comparison of 5-year cardiovascular event rates by living arrangement status

\begin{tabular}{|c|c|c|c|c|c|}
\hline \multirow[b]{2}{*}{ Outcome } & \multicolumn{2}{|c|}{5 -year event rate $(\%)$} & \multicolumn{3}{|c|}{ Time to first event $\mathrm{HR}(95 \% \mathrm{Cl})$} \\
\hline & $\begin{array}{l}\text { Living alone } \\
(\mathrm{n}=3648)\end{array}$ & $\begin{array}{l}\text { Not living alone } \\
(\mathrm{n}=28728)\end{array}$ & Unadjusted & Adjusted (age and sex) & Adjusted $^{*}$ \\
\hline MACE & 10.3 & 8.5 & 1.24 (1.11 to 1.38$), p<0.001$ & 1.11 (0.99 to 1.24$), p=0.07$ & 1.04 (0.92 to 1.18$), p=0.52$ \\
\hline All-cause death & 9.8 & 7.6 & 1.31 (1.17 to 1.47$), p<0.001$ & 1.13 (1.01 to 1.26$), p=0.04$ & $1.08(0.95$ to 1.23$), p=0.25$ \\
\hline CV death & 6.5 & 4.8 & 1.37 (1.20 to 1.58$), p<0.001$ & 1.18 (1.02 to 1.35$), p=0.02$ & 1.12 (0.95 to 1.32$), p=0.17$ \\
\hline MI & 3.5 & 3.4 & 1.04 (0.86 to 1.25$), p=0.70$ & 1.01 (0.84 to 1.22$), p=0.93$ & 0.97 (0.78 to 1.19$), p=0.76$ \\
\hline Stroke & 2.7 & 2.0 & 1.35 (1.09 to 1.67$), p=0.006$ & 1.15 (0.93 to 1.43$), p=0.20$ & $1.00(0.77$ to 1.30$), p=0.99$ \\
\hline Unstable angina & 10.8 & 11.0 & 0.99 (0.89 to 1.10$), p=0.86$ & 0.97 (0.87 to 1.08$), p=0.55$ & 0.99 (0.88 to 1.12$), p=0.91$ \\
\hline Major bleeding & 1.4 & 1.4 & 1.01 (0.75 to 1.35$), p=0.96$ & $0.90(0.67$ to 1.21$), p=0.48$ & 0.91 (0.66 to 1.24$), p=0.55$ \\
\hline Hospitalisation for CHF & 5.6 & 5.2 & 1.10 (0.95 to 1.28$), p=0.20$ & 1.02 (0.87 to 1.18$), p=0.83$ & 1.07 (0.89 to 1.27$), p=0.48$ \\
\hline
\end{tabular}

social support and its impact on both health-related quality of life, as well as psychological and physical function. ${ }^{21}$ The difference in CV risk factors between women living alone and women living with others has not been clearly defined; women living alone may have an increased risk of developing diabetes, are more likely to smoke and less likely to have hypertension. ${ }^{1922}$ In the current era, women are more financially and socially independent than previous generations, with technology enhancing accessibility and communication. It may be that living status in women may not be as strong a reflection of social isolation compared with males. These suggestions are speculative as previous studies have failed to show this sex-based interaction identified in the CLARIFY registry. ${ }^{5} 13 \quad 17 \quad 18 \quad 23$ Further studies are warranted to assess factors that may attribute to the difference in CV risk in men and women living alone such as location of residence (urban or rural), social supports, social networks, local healthcare resources, as well as institutionalisation, marital status and progression of $\mathrm{CV}$ risk factors, which may guide novel interventions to reduce recurrent $\mathrm{CV}$ events in men living alone.

Living Alone Not Living Alone Hazard Ratio (95\% Cl); P-value Interaction P-Value

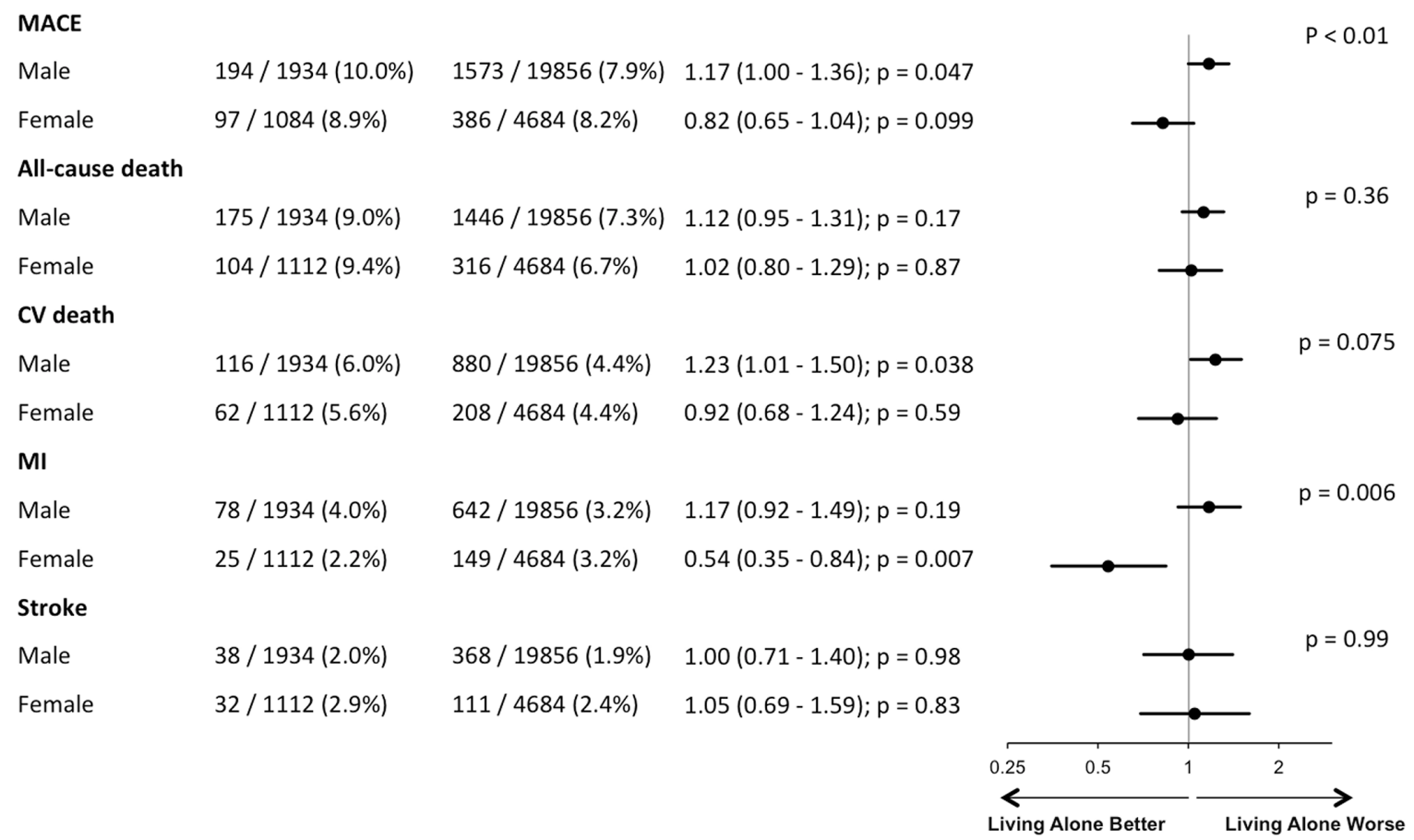

Figure 1 Forest plot: event rates, adjusted HRs and multivariate interaction by sex according to living arrangement status 5-year event rate and HRs for the primary endpoint of MACE and secondary endpoints (all-cause death, CV death, MI and stroke) stratified by sex according to living arrangement status. Adjusted HRs associated with living alone compared with living with others (reference). The multivariate analysis was adjusted for age, sex, geographical region, smoking status, diabetes, peripheral arterial disease, MI, percutaneous coronary intervention, coronary artery bypass graft surgery, asthma/chronic obstructive pulmonary disease, congestive heart failure, systolic blood pressure, diastolic blood pressure, left ventricular ejection fraction and number of vessels with coronary artery stenosis. CV, cardiovascular; MACE, major adverse cardiovascular event; MI, myocardial infarction. 
Living Alone

MACE

$<65$ years

$65-74$ years

$\geq 75$ years

All-cause death

$<65$ years

$65-74$ years

$\geq 75$ years

$\mathrm{CV}$ death

$<65$ years

$65-74$ years

$\geq 75$ years

MI

$<65$ years

$65-74$ years

$\geq 75$ years

\section{Stroke}

$<65$ years

$65-74$ years

$\geq 75$ years
Not Living Alone

Hazard Ratio (95\% Cl); P-value

$730 / 12960(5.6 \%) \quad 1.21(0.96-1.52) ; p=0.10$

$707 / 8025$ (8.8\%) $0.97(0.78-1.21) ; p=0.81$

$522 / 3555(14.7 \%) \quad 0.99(0.80-1.23) ; p=0.92$

112 / 758 (14.8\%)

$48 / 1204(4.0 \%)$

$103 / 1084$ (9.5\%)

$128 / 758(16.9 \%)$

34 / 1204 (2.8\%)

$58 / 1084(5.4 \%)$

$86 / 758(11.3 \%)$

47 / 1204 (3.9\%)

$31 / 1084(2.9 \%)$

$25 / 758(3.3 \%)$

$20 / 1204(1.7 \%)$

32 / 1084 (3.0\%)

$18 / 758(2.4 \%)$
$497 / 12960(3.8 \%) \quad 1.03(0.76-1.38) ; p=0.87$

$687 / 8025(8.6 \%) \quad 1.10(0.89-1.37) ; p=0.36$

$578 / 3555(16.3 \%) \quad 1.09(0.89-1.34) ; p=0.40$

$328 / 12960(2.5 \%) \quad 1.11(0.78-1.59) ; p=0.56$

$398 / 8025(5.0 \%) \quad 1.07(0.81-1.43) ; p=0.62$

$362 / 3555(10.2 \%) \quad 1.17(0.91-1.50) ; p=0.22$

$371 / 12960(2.9 \%) \quad 1.31(0.96-1.78) ; p=0.088$

$269 / 8025$ (3.4\%) $0.83(0.57-1.21) ; p=0.33$

$151 / 3555$ (4.2\%) $0.67(0.43-1.04) ; p=0.077$

$164 / 12960(1.3 \%) \quad 1.34(0.84-2.14) ; p=0.22$

$185 / 8025(2.3 \%) \quad 1.14(0.78-1.69) ; p=0.50$

$130 / 3555(3.7 \%) \quad 0.66(0.39-1.10) ; p=0.11$
Interaction P-Value

$p=0.33$

$p=0.93$

$p=0.88$

$p=0.046$

Figure 2 Forest plot: event rates, adjusted HRs and multivariate interaction by age group according to living arrangement status 5-year event rate and HRs for the primary endpoint of MACE and secondary endpoints (all-cause death, CV death, MI and stroke) stratified by age group according to living arrangement status. Adjusted HRs associated with living alone compared with living with others (reference). The multivariate analysis was adjusted for age, sex, geographical region, smoking status, diabetes, peripheral arterial disease, $\mathrm{MI}$, percutaneous coronary intervention, coronary artery bypass graft surgery, asthma/chronic obstructive pulmonary disease, congestive heart failure, systolic blood pressure, diastolic blood pressure, left ventricular ejection fraction number of vessels with coronary artery stenosis. CV, cardiovascular; MACE, major adverse cardiovascular event; $\mathrm{MI}$, myocardial infarction.

Similar to findings from the REACH registry, we observed trends towards a lower risk of recurrent MI and stroke among elderly patients ( $\geq 75$ years) living alone, while younger patients ( $<65$ years) living alone had increased risk. Patients $<65$ years may have relatively complex social interactions with increased stress, anxiety and depression resulting in poor health behaviour with adverse haemodynamic effects that may lead to the progression of CVD. Elderly patients $\geq 75$ years living alone may reflect those who have less comorbid conditions and are able to live independently and do not require assisted living or nursing home level of care. Discharge planning in elderly patients is multifaceted with a focus on mobility, home safety and methods to improve medication adherence with early and close outpatient follow-up. Lower CV events in elderly patients living alone may also represent the success of postdischarge initiatives in this patient population. Our analysis suggests that there is not an association between elderly women living alone and increased risk for $\mathrm{CV}$ events but there may be in men; this finding is hypothesis generating, and further analyses are needed to elucidate the potential mechanisms underlying this difference.

With advancements in the management CAD, patients are living longer with improved health and functional status. For this reason, living situation is an important variable to account for, and thus our analysis has several important implications. After adjustment, we did not identify that living alone is associated with an independent risk for MACE in patients with stable CAD. There was similar use of guideline recommended therapy in both living status groups. This is in contrast to previous studies that suggest that patients living alone may be at increased risk of CV events. This may represent an improvement of care provided by clinicians managing these patients with increased long-term awareness of psychosocial issues, ensuring optimal care is delivered, potentially limiting the care gap in this patient population. Similarly, the suggested lower risk in elderly patients living alone may be a testament to close clinical follow-up and adequacy of social supports provided that allows these individuals to remain living alone in the community without institutionalisation. The novel finding of potential lower CV risk in women warrants further assessment. Women living alone may have coping mechanisms that we were not able to identify in our analysis that may have accounted for this difference. As CV specialists caring for patients with established CAD, it is important to consider psychosocial factors such as living status, which may increase CV risk. Physicians should counsel patients to report symptoms immediately without delay to medical attention and identify those that may benefit from further psychosocial intervention. Future 


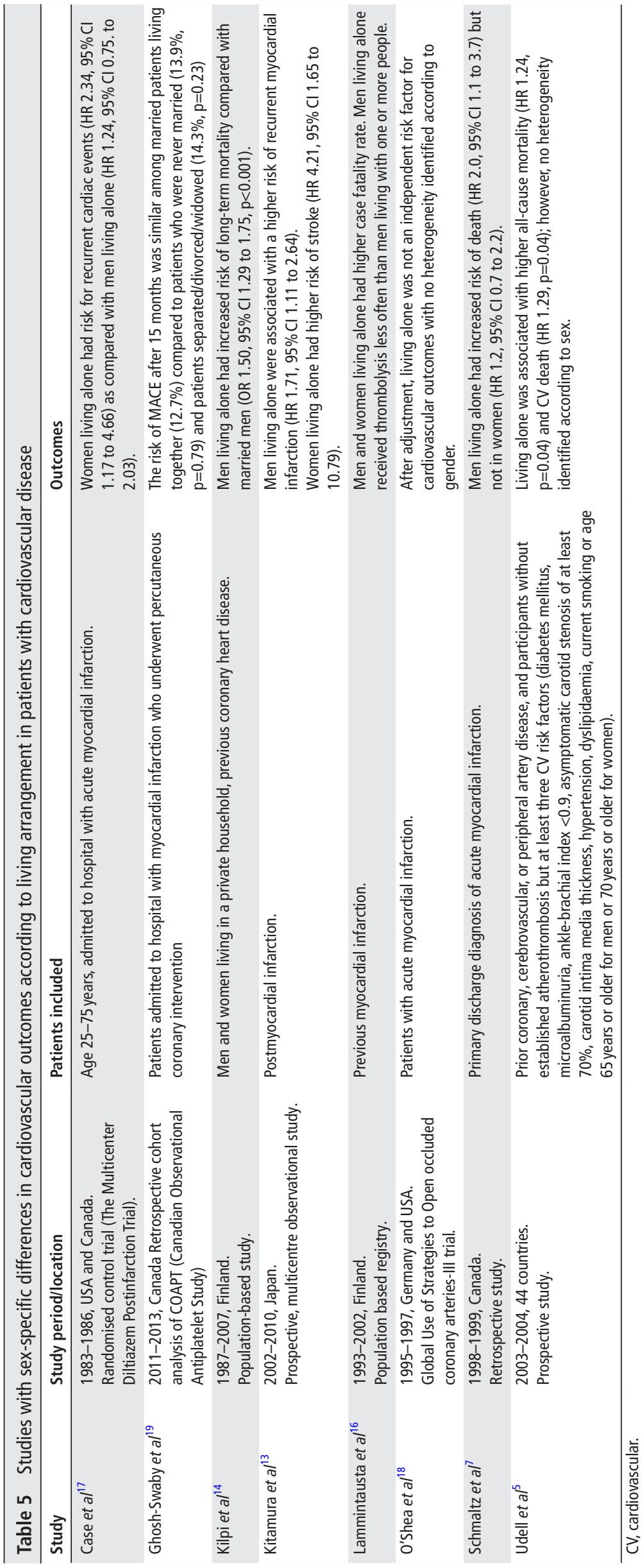


studies can explore the role of interventional social support, such as cardiac rehabilitation, which may improve outcomes in these subgroups. To date, there is little trial data to show providing multidisciplinary social support improves outcomes following a cardiac event, but several years have passed and new methods of connectivity now exist that may improve effects.

There are several limitations to our analysis. The case report form identified patients living alone at baseline, which we used as a marker of social isolation. Specific details were not provided about living status including living conditions (type of residence) and proximity of social supports and resources, which may have further differentiated this heterogeneous population. In addition, we were not able to account or adjust for unidentified confounders such as stress, depression and socioeconomic status. Unfortunately, this information is not available, and although we tried to adjust for various patient characteristics, we acknowledge that there remains a potential for residual confounding. Physicians investigators completed the electronic case report form at baseline and yearly entering the patient information. It is unclear if the physician investigator contacted the patient directly or via relatives. Furthermore, study endpoints were determined by the site investigators without central adjudication. A previous study in young patients with MI demonstrated those with lower social support were more likely to live alone but also had poorer mental health status, quality of life scores and more depression. ${ }^{24}$ Further exploration between living status and mental health outcomes may give insight into this complex interaction. Patients excluded from the CLARIFY registry included those with serious non-CVD or conditions interfering with life expectancy (ie, cancer and drug abuse) or severe CVD such as advanced heart failure and were less likely to live alone; the impact this may have had on the analysis is speculative. Our case report form did not identify marital status, which may have given further insight into the heterogeneity of the risk of living alone in women and different age groups. For elderly patients, we were not able to use any other markers of functional status, which may have given further insight into CV risk. Living status was recorded only at baseline, and we were not able to describe how long each patient was living alone or account for the need

\section{Key messages}

What is already known on this subject?

- Patients living alone with coronary artery disease (CAD) may be at increased risk for cardiovascular (CV) events. As individuals with stable CAD live longer with advanced comorbidities, there is an important public health implication to determine if living status is independently associated with poor health outcomes.

\section{What might this study add?}

- The prospeCtive observational LongitudinAl Reglstry of patients with stable coronarY artery disease registry included outpatients with CAD with 5 years of follow-up. Our study suggests that living alone in patients with stable CAD was not associated with an independent increase in major adverse cardiovascular events, although age-based and sex-based differences were apparent.

\section{How might this impact on clinical practice?}

- Elderly patients and women living alone may have potentially lower CV risk, which warrants further confirmation. for change in living status throughout the follow-up period. The case report form only identified clinical endpoints as described; we were not able to account for other markers of healthcare consumption such as hospitalisation or increased utilisation of local health services.

\section{CONCLUSION}

Living alone in patients with stable CAD was not associated with an independent increase in MACE, although age and sex-based differences were apparent. Elderly patients and women living alone may have potentially lower CV risk. Future large studies in patients with established CAD should further evaluate key components of social isolation using validated in-depth tools/ scoring systems. This will better inform us of higher risk components of social deprivation that will help guide potential psychosocial interventions.

\section{Author affiliations}

${ }^{1}$ Terrence Donnelly Heart Centre, St Michael's Hospital, University of Toronto, Toronto, Ontario, Canada

${ }^{2}$ Robertson Centre for Biostatistics, University of Glasgow, Glasgow, United Kingdom ${ }^{3}$ Department of Cardiology and LTTA Centre, University Hospital of Ferrara and Maria Cecilia Hospital, Cotignola, Italy

${ }^{4}$ Severance Cardiovascular Hospital, Yonsei College of Medicine, Seoul, South Korea ${ }^{5}$ Jefe de Cardiología Intervencionista, Universidad Autónoma de Querétaro, Querétaro, Mexico

${ }^{6}$ Institute of Cardiovascular Medicine and Science, Royal Brompton Hospital, National Heart and Lung Institute, Imperial College, London, United Kingdom

${ }^{7}$ Montreal Heart Institute, Université de Montréal, Montreal, Quebec, Canada

${ }^{8}$ Medical University ofSilesia, School of Medicine in Katowice, Katowice, Poland

${ }^{9}$ French Alliance for Cardiovascular Trials, an F-CRIN network, Université Paris-Didero, AP-HP and INSERM U1148, Paris, France

${ }^{10}$ Peter Munk Cardiac Centre and Women's College Hospital, University of Toronto, Toronto, Ontario, Canada

Acknowledgements The authors gratefully acknowledge the contributions of the TheprospeCtive observational LongitudinAl Reglstry of patients with stablecoronarY artery disease (CLARIFY) patients, investigators, research coordinators and committee members.

Contributors IF had full access to all the data in the study and takes responsibility for the integrity of the data and the accuracy of the data analysis. SG wrote the first draft of the manuscript. Study conception or design: SG, SGG, GS and JAU. Acquisition, analysis or interpretation of data: all authors. Critical revision of the manuscript for important intellectual content: all authors. Statistical analysis: IF, NG and PM. Administrative, technical and material support: SGG, NG and GS. Study supervision: SGG and GS. All authors have approved the final article.

Funding The CLARIFY registry is supported by Servier. The sponsor assisted with the set-up, data collection and management of the study in each country.

Competing interests All authors have completed the ICMJE uniform disclosure form at www.icmje.org/coi_disclosure.pdf and declare: JAU reported consultancy fees from consulting: Johnson \& Johnson, Merck, Novartis, Sanofi Pasteur; Novartis (steering committee). PD reports grants and personal fees from Servier, outside the submitted work. SGG reports grants from Servier during the conduct of the study; personal fees from Servier Canada, outside the submitted work. RF reports honorarium from Servier for steering committee membership, consulting, speaker's bureau fees and support for travel to study meetings; personal fees from BoehringerIngelheim, Novartis, Merck Serono and Irbtech; he is a stockholder in Medical Trials Analysis. IF reports grants and personal fees from Servier during the conduct of the study; grants and personal fees from Amgen, outside the submitted work. KMF reports personal fees and non-financial support from Servier during the conduct of the study, from Broadview Ventures; personal fees from AstraZeneca, TaurX, CelAegis, outside the submitted work; non-financial support from Armgo, Director of Vesalius Trials LtdDinimal and stockholder of Armgo and CellAegis. NG reports grants from Servier during the conduct of the study. PMS reports grants from Servier during the conduct of the study. PGS reports grants from Merck, Sanofi, Servier; personal fees from Amarin, Amgen, AstraZeneca, Bayer, Boehriner-Ingelheim, BristolMyersSquibb, CSL-Behring, Daiichi-Sankyo, Janssen, Lilly, Merck, Novartis, Pfizer, Regeneron, Sanofi, Servier, The Medicines Company, outside the submitted work. JCT reports personal fees from Servier, during the conduct of the study; grants from Amarin, Astra-Zeneca, DalCor, Eli Lilly, Esperion, Ionis, Merck, Pfizer, Sanofi, Servier; personal fees from DalCor, Pfizer, Sanofi, Servier, holds minor equity interest in DalCor, outside the submitted work; a patent Pharmacogenomics-Guided 
Therapy with CETP Inhibitor pending. MT reports personal fees from Servier during the conduct of the study; personal fees from Bayer, Celyad, KOWA, Janssen-Cilag, PERFUSE Study Group; grants from Polish National Center for Research and Development, outside the submitted work.

Patient consent for publication Not required.

Ethics approval The study was approved by the National Research Ethics Service, Isle of Wight, Portsmouth and Southeast Hampshire Research Ethics Committee, UK.

Provenance and peer review Not commissioned; externally peer reviewed.

Data sharing statement The corresponding author had full access to all the data in the study and had final responsibility for the decision to submit for publication.

\section{REFERENCES}

1 Rozanski A, Blumenthal JA, Kaplan J. Impact of psychological factors on the pathogenesis of cardiovascular disease and implications for therapy. Circulation 1999:99:2192-217.

2 Clark AM, DesMeules M, Luo W, et al. Socioeconomic status and cardiovascular disease: risks and implications for care. Nat Rev Cardiol 2009;6:712-22.

3 Blumenthal JA, Sherwood A, Smith PJ, et al. Enhancing Cardiac Rehabilitation With Stress Management Training: A Randomized, Clinical Efficacy Trial. Circulation 2016;133:1341-50.

4 Holt-Lunstad J, Smith TB, Layton JB. Social relationships and mortality risk: a metaanalytic review. PLoS Med 2010;7:e1000316.

5 Udell JA, Steg PG, Scirica BM, et al. Living alone and cardiovascular risk in outpatients at risk of or with atherothrombosis. Arch Intern Med 2012;172:1086-95.

6 Nakatsuma K, Shiomi $\mathrm{H}$, Watanabe $\mathrm{H}$, et al. Comparison of long-term mortality after acute myocardial infarction treated by percutaneous coronary intervention in patients living alone versus not living alone at the time of hospitalization. Am J Cardiol 2014;114:522-7.

7 Schmaltz HN, Southern D, Ghali WA, et al. Living alone, patient sex and mortality after acute myocardial infarction. J Gen Intern Med 2007;22:572-8.

8 Yusuf $S$, Hawken S, Ounpuu S, et al. Effect of potentially modifiable risk factors associated with myocardial infarction in 52 countries (the INTERHEART study): casecontrol study. Lancet 2004;364:937-52.

9 Gandhi S, Dorian P, Greenlaw N, et al. Characteristics and evidence-based management of stable coronary artery disease patients in Canada compared with the rest of the world: insights from the CLARIFY registry. Can J Cardiol 2014;30:132-7.

10 Steg PG. Heart rate management in coronary artery disease: the CLARIFY registry. European Heart Journal Supplements 2009;11;(suppl D):D13-18.
11 Steg PG, Ferrari R, Ford I, et al. Heart rate and use of beta-blockers in stable outpatients with coronary artery disease. PLoS One 2012;7:e36284.

12 Steg PG, Greenlaw N, Tardif JC, et al. Women and men with stable coronary artery disease have similar clinical outcomes: insights from the international prospective CLARIFY registry. Eur Heart J 2012;33:2831-40.

13 Kitamura T, Sakata Y, Nakatani D, et al. Living alone and risk of cardiovascular events following discharge after acute myocardial infarction in Japan. J Cardiol 2013:62:257-62.

14 Kilpi $\mathrm{F}$, Konttinen $\mathrm{H}$, Silventoinen $\mathrm{K}$, et al. Living arrangements as determinants of myocardial infarction incidence and survival: A prospective register study of over 300,000 Finnish men and women. Soc Sci Med 2015;133:93-100.

15 Redfors $\mathrm{P}$, Isaksén $\mathrm{D}$, Lappas $\mathrm{G}$, et al. Living alone predicts mortality in patients with ischemic stroke before 70 years of age: a long-term prospective follow-up study. BMC Neurol 2016;16:80.

16 Lammintausta A, Airaksinen JK, Immonen-Räihä P, et al. Prognosis of acute coronary events is worse in patients living alone: the FINAMI myocardial infarction register. Eur J Prev Cardiol 2014;21:989-96.

17 Case RB, Moss AJ, Case N, et al. Living alone after myocardial infarction. Impact on prognosis. JAMA 1992;267:515-9.

18 O'Shea JC, Wilcox RG, Skene AM, et al. Comparison of outcomes of patients with myocardial infarction when living alone versus those not living alone. Am J Cardiol 2002;90:1374-7.

19 Ghosh-Swaby OR, Tan M, Bagai A, et al. Marital status and outcomes after myocardial infarction: Observations from the Canadian Observational Antiplatelet Study (COAPT). Clin Cardiol 2018;41:285-92.

20 Kandler U, Meisinger C, Baumert J, et al. Living alone is a risk factor for mortality in men but not women from the general population: a prospective cohort study. BMC Public Health 2007;7:335.

21 Staniute M, Brozaitiene J, Bunevicius R. Effects of social support and stressful life events on health-related quality of life in coronary artery disease patients. J Cardiovasc Nurs 2013;28:83-9.

22 Lidfeldt J, Nerbrand C, Samsioe G, et al. Women living alone have an increased risk to develop diabetes, which is explained mainly by lifestyle factors. Diabetes Care 2005;28:2531-6

23 Norekvål TM, Fridlund B, Moons $\mathrm{P}$, et al. Sense of coherence-a determinant of quality of life over time in older female acute myocardial infarction survivors. J Clin Nurs 2010;19(5-6):820-31.

24 Bucholz EM, Strait KM, Dreyer RP, et al. Effect of low perceived social support on health outcomes in young patients with acute myocardial infarction: results from the VIRGO (Variation in Recovery: Role of Gender on Outcomes of Young AMI Patients) study. J Am Heart Assoc 2014;3:e001252. 